\title{
p38-MAP Kinase Negatively Regulates the Slow Force Response to Stretch in Rat Myocardium through the Up-Regulation of Dual Specificity Phosphatase 6 (DUSP6)
}

\author{
Maite R. Zavala Romina G. Díaz Andrés J. Medina María P. Acosta \\ Daiana S. Escudero Irene L. Ennis Néstor G. Pérez María C. Villa-Abrille \\ Centro de Investigaciones Cardiovasculares "Dr. Horacio E. Cingolani", CONICET La Plata, Facultad de \\ Ciencias Médicas, Universidad Nacional de La Plata, La Plata, Argentina
}

\section{Key Words}

$\mathrm{NHE}-1 \cdot \mathrm{SFR} \cdot \mathrm{p} 38-\mathrm{MAPK} \cdot$ Stretch $\cdot \mathrm{DUSP} 6$

\begin{abstract}
Background/Aims: Myocardial stretch increases cardiac force in two consecutive phases: The first one due to Frank-Starling mechanism, followed by the gradually developed slow force response (SFR). The latter is the mechanical counterpart of an autocrine/paracrine mechanism involving the release of angiotensin II (Ang II) and endothelin (ET) leading to $\mathrm{Na}^{+} / \mathrm{H}^{+}$exchanger 1 (NHE-1) phosphorylation and activation. Since previous evidence indicates that p38-MAP kinase (p38-MAPK) negatively regulates the Ang II-induced NHE1 activation in vascular smooth muscle and the positive inotropic effect of ET in the heart, we hypothesized that this kinase might modulate the magnitude of the SFR to stretch. Methods: Experiments were performed in isolated rat papillary muscles subjected to sudden stretch from 92 to $98 \%$ of its maximal length, in the absence or presence of the p38-MAPK inhibitor SB202190, or its inactive analogous SB202474. Western blot technique was used to determine phosphorylation level of p38-MAPK, ERK1/2, p90RSK and NHE-1 (previously immunoprecipitated with NHE-1 polyclonal antibody). Dual specificity phosphatase 6 (DUSP6) expression was evaluated by RT-PCR and western blot. Additionally, the $\mathrm{Na}^{+}$-dependent intracellular $\mathrm{pH}$ recovery from an ammonium prepulse-induced acid load was used to asses NHE-1 activity. Results: The SFR was larger under p38-MAPK inhibition (SB202190), effect that was not observed in the presence of an inactive analogous (SB202474). Myocardial stretch activated p38-MAPK, while pre-treatment with SB202190 precluded this effect. Inhibition of p38-MAPK increased stretched-induced NHE-1 phosphorylation and activity, key event in the SFR development. Consistently, p38-MAPK inhibition promoted a greater increase in ERK1/2-p90RSK phosphorylation/activation after myocardial stretch, effect that may certainly be responsible for the observed increase in NHE-1 phosphorylation under this condition.

M. R. Zavala and R. G. Díaz contributed equally to this work.




\section{Cellular Physiology Cell Physiol Biochem 2019;52:172-185 \\ \begin{tabular}{ll|l} 
and Biochemistry & $\begin{array}{l}\text { DOl: 10.33594/000000012 } \\
\text { Published online: } 28 \text { February } 2019\end{array}$ & $\begin{array}{l}\text { O } 2019 \text { The Author(s). Published by } \\
\text { Cell Physiol Biochem Press GmbH\&Co. KG }\end{array}$ \\
\cline { 2 - 3 }
\end{tabular} \\ Zavala et al.: Myocardial Stretch and p38-MAPK}

Myocardial stretch induced up-regulation of the DUSP6, which specifically dephosphorylates ERK1/2, effect that was blunted by SB202190. Conclusion: Taken together, our data support the notion that p38-MAPK activation after myocardial stretch restricts the SFR by limiting ERK1/2-p90RSK phosphorylation, and consequently NHE-1 phosphorylation/activity, through a mechanism that involves DUSP6 up-regulation.

(C) 2019 The Author(s). Published by Cell Physiol Biochem Press GmbH\&Co. KG

\section{Introduction}

Myocardial stretch is an important physiological stimulus that allows the heart to modulate its contractile performance, and consequently adapt cardiac output to changes in hemodynamic conditions. It is well known that the stretch of cardiac muscle induces a two-phase increase in force: First, the Frank-Starling mechanism attributed to enhanced myofilament responsiveness to calcium; followed by the slow force response (SFR), due to an increase in the intracellular calcium transient. Previous studies from our laboratory provided evidence supporting that the SFR is the mechanical expression of a stretch-triggered autocrine/paracrine loop of cell signaling that involves: 1) the sequential activation of angiotensin II (Ang II), endothelin (ET) and mineralocorticoid receptors (AT1, ETA and MR respectively), 2) transactivation of the epidermal growth factor receptor, 3) reactive oxygen species (ROS) production, 5) activation of kinases upstream the cardiac isoform of the $\mathrm{Na}^{+}$/ $\mathrm{H}^{+}$exchanger (NHE-1), such as ERK1/2 and p90RSK, 6) NHE-1 hyperactivity, 7) increase in intracellular $\mathrm{Na}^{+}$concentration, and 8) increase in $\mathrm{Ca}^{2+}$ transient amplitude through reverse $\mathrm{Na}^{+} / \mathrm{Ca}^{2+}$ exchange [1]. In the present study we explored the possible participation of p38 MAP kinase (p38-MAPK) in this intracellular cascade. Previous evidence highlights the importance of p38-MAPK activation in the regulation of different cardiovascular processes [2-4]. In this sense, even though several reports showed that p38-MAPK negatively regulates cardiac function [5-9], the underlying mechanism is not fully understood. It has been shown that inhibition of p38-MAPK increases myofilament $\mathrm{Ca}^{2+}$ sensitivity [5], and its activation decreases $\alpha$-tropomyosin phosphorylation and ATPase activity [9]. Interestingly, Szokodi et al. [10] working in isolated perfused rat hearts showed that pharmacological inhibition of p38-MAPK strengthens the positive inotropic response to ET-1, effect attributed to an increased phosphorylation of phospholamban (PLN) at the residue serine 16 . On the other hand, experiments by Kusuhara et al. [11] in isolated vascular smooth muscles cells showed that Ang II promotes not only ERK1/2 but also p38-MAPK activation. Interestingly, the authors proposed a crosstalk between these kinases, being p38-MAPK responsible for a decrease in ERK1/2 activity that consequently reduces the activation of its downstream kinase, p90RSK. Later on, this group showed in the same preparation that p90RSK was able to phosphorylate the serine 703 of the NHE-1 stimulating its activity in response to Ang II [12]. They coined the term "NHE-1 kinase" to identify the p90RSK [12]. Taken this evidence together, and considering that Ang II, ET and the NHE-1 are known mediators of the signaling pathway leading to the SFR, we hypothesized that p38-MAPK may conceivably participate in this important mechanism. In this work, we will present evidence supporting that p38-MAPK activation after myocardial stretch limits the increase in force during the SFR by limiting NHE-1 phosphorylation through a mechanism that involves up-regulation of the dual specificity phosphatase 6 (DUSP6).

\section{Materials and Methods}

All procedures followed during this investigation conform to the Guide for the Care and Use of Laboratory Animals (National Research Council. 2011, Eighth Edition. Washington, DC: The National Academies Press) [13] and the experimental protocol was approved by the Animal Welfare Committee of La Plata School of Medicine. 


\section{Cellular Physiology Cell Physiol Biochem 2019;52:172-185 \\ \begin{tabular}{ll|l} 
and Biochemistry & $\begin{array}{l}\text { DOl: 10.33594/000000012 } \\
\text { Published online: } 28 \text { February } 2019\end{array}$ & $\begin{array}{l}\text { O } 2019 \text { The Author(s). Published by } \\
\text { Cell Physiol Biochem Press GmbH\&Co. KG }\end{array}$ \\
\cline { 2 - 3 }
\end{tabular} \\ Zavala et al.: Myocardial Stretch and p38-MAPK}

Wistar rats ( $\mathrm{n}=36$, bodyweight 300-400 g) were anaesthetized by intraperitoneal injection of sodium pentobarbital ( $35 \mathrm{mg} / \mathrm{kg}$ body weight). The chests were opened to excise the heart when deep anaesthesia was reached, verified by the loss of corneal reflex and appearance of slow deep diaphragmatic breathing. Animals were provided by the Animal Facilities of the Centro de Investigaciones Cardiovasculares, "Dr. Horacio E. Cingolani". Each heart was used to provide at least 2 papillary muscles.

\section{Isolation of papillary muscles}

Papillary muscles from the left ventricle were used to assess the SFR to stretch as previously described [14]. Briefly, the muscles were mounted in a perfusion chamber placed on the stage of an inverted microscope (Olympus) and superfused at a constant rate $(5 \mathrm{ml} / \mathrm{min})$ with a $\mathrm{CO}_{2} / \mathrm{HCO}_{3}{ }^{-}$-buffered solution containing (mmol/L): $\mathrm{NaCl} 128.3, \mathrm{KCl} 4.5, \mathrm{CaCl}_{2} 1.35, \mathrm{NaHCO}_{3} 20.23, \mathrm{MgSO}_{4} 1.05$, glucose 11.0 and equilibrated with 5 $\% \mathrm{CO}_{2}-95 \% \mathrm{O}_{2}(\mathrm{pH} \sim 7.40)$. The possible participation of catecholamines released by the nerve endings was prevented by adrenergic receptors blockade with $1.0 \mu \mathrm{mol} / \mathrm{L}$ prazosin plus $1.0 \mu \mathrm{mol} / \mathrm{L}$ atenolol. The muscles were paced at $0.2 \mathrm{~Hz}$ at a voltage $10 \%$ over threshold maintained at $30^{\circ} \mathrm{C}$, and isometric contractions were recorded. Cross sectional area of each muscle (CSA in $\mathrm{mm}^{2}$ : Stretch 0.356 $\pm 0.026, \mathrm{n}=16$; Stretch+SB202190 $0.399 \pm 0.024, \mathrm{n}=21$; Stretch+SB202474 0.369 $\pm 0.051, \mathrm{n}=8$; NS), which was calculated as 0.75 of the product of thickness and width, was used to normalize force records obtained with a silicon strain gauge (model AEM 801, Kronex Technologies Corp, Oakland, CA, USA). Immediately after mounting, the slack length of each muscle was determined and then the muscles were progressively stretched to the length at which they developed maximal twitch force ( $L$ max). After a few minutes at $L$ max, they were shortened to obtain the $95 \%$ of the maximal twitch force (length that approximated $98 \%$ of $L$ max and referred to as $L 98$ ). Then, the muscles were shortened to $92 \%$ of $L \max (L 92)$ and maintained at this length until the beginning of the experimental protocol, when they were abruptly stretched from $L 92$ to L98. p38-MAPK inhibitor, SB202190, or its inactive analogous, SB202474, were added to the perfusate 20 min before stretch. Neither the p38-MAPK inhibitor, SB202190, nor its inactive analogous, SB202474, induced significant changes in basal developed force of papillary muscles $(101.9 \pm 1.2 \%$ of pre-SB202190, n=21 and $104.2 \pm 1.8 \%$ of preSB202474, n=8).

\section{NHE-1 activity}

Intracellular $\mathrm{pH}\left(\mathrm{pH}_{\mathrm{i}}\right)$ was measured in isolated left ventricular papillary muscles from male Wistar rats following a previously described BCECF-epifluorescence technique [15]. The muscles were loaded with the ester form of the pH-sensitive fluorescent dye BCECF (Invitrogen, Molecular Probes). The dye was solubilized in dimethyl sulfoxide (Sigma Chemical Co) containing pluronic acid F-127 (20\% [wt/vol]) and diluted to a final concentration of $10 \mu \mathrm{mol} / \mathrm{L}$ in $\mathrm{HCO}_{3}-/ \mathrm{CO}_{2}$-buffered solution. After autofluorescence levels were recorded, the muscles were incubated for 1 hour in the BCECF-AM containing solution. At the end of the loading period, washout of the extracellular space with dye-free solution was carried out for 30-60 minutes before any $\mathrm{pH}_{\mathrm{i}}$ determination was done. At this time, BCECF fluorescence was uniformly distributed throughout the muscle. To measure fluorescence emission from BCECF, excitation light from a 75-W Xenon lamp was band pass-filtered alternatively at 440 and $495 \mathrm{~nm}$ and was then transmitted to the muscles under study by a dichroic mirror (reflecting wavelengths, pass filter at $535 \pm 5 \mathrm{~nm}$ to a photomultiplier (model R2693, Hamamatsu). The output of the photomultiplier was collected via an A/D converter (model 2801 A, Data Translation) and stored in a personal computer for later analysis. To limit photobleaching, a neutral density filter ( $1 \%$ transmittance) was placed in the excitation light path, and a manual shutter was used to select sampling intervals ( 2 seconds every 10 seconds) during the protocol.

The NHE-1 activity, after sustained acidosis, was assessed by the $\mathrm{Na}^{+}$-dependent $\mathrm{pH}_{\mathrm{i}}$ recovery from an ammonium prepulse-induced acute acid load [15]. The experiments were performed in the nominal absence of bicarbonate (HEPES buffer) to assure that the $\mathrm{pH}_{\mathrm{i}}$ recovery after the acidic load was entirely due to NHE-1 activation. The papillary muscles were acid loaded by brief (10 min) exposure to $20.0 \mathrm{mmol} / \mathrm{L}$ $\mathrm{NH}_{4} \mathrm{Cl}$ followed by washout with $\mathrm{Na}^{+}$-free HEPES buffer $(\mathrm{NaCl}$ was equimolar replaced with $\mathrm{N}$-methylD-glucamine). Recovery of $\mathrm{pH}_{\mathrm{i}}$ after acid loading was induced by re-introduction of external $\mathrm{Na}^{+}$after 10 minutes of acidosis. The intracellular buffer capacity ( $\beta \mathrm{i}$ ) was determined as previously described [16]. Maximal $\mathrm{H}^{+}$efflux $\left(\mathrm{J}_{\mathrm{H}}{ }^{+}={ }_{\max } \mathrm{dpH}_{\mathrm{i}} / \mathrm{dt}\right.$. $\left.\beta_{\mathrm{i}}\right)$ was calculated at the beginning of recovery from acidosis (just after 


\section{Cellular Physiology Cell Physiol Biochem 2019;52:172-185 \\ \begin{tabular}{ll|l} 
and Biochemistry & $\begin{array}{l}\text { DOl: 10.33594/000000012 } \\
\text { Published online: } 28 \text { February } 2019\end{array}$ & $\begin{array}{l}\text { O } 2019 \text { The Author(s). Published by } \\
\text { Cell Physiol Biochem Press GmbH\&Co. KG }\end{array}$ \\
\cline { 2 - 3 } & Zave
\end{tabular} \\ Zavala et al.: Myocardial Stretch and p38-MAPK}

extracellular $\mathrm{Na}^{+}$re-addition) and used to compare NHE-1 activity among different groups. The maximal intracellular acidification after the acidic load (measured just before $\mathrm{Na}^{+}$re-addition) was of similar magnitude in both experimental groups.

At the end of each experiment, fluorescence emission was calibrated by exposing the muscles to a high$\mathrm{KCl}$ solution containing $10 \mu \mathrm{mol} / \mathrm{L}$ nigericin, a $\mathrm{H}^{+}-\mathrm{K}^{+}$exchanger that equals $\left[\mathrm{H}^{+}\right]_{0}$ to $\left[\mathrm{H}^{+}\right]_{\mathrm{i}}$ when extracellular and intracellular $\mathrm{K}^{+}$are the same. The calibration solution contained (in mmol/L) $\mathrm{KCl} 140.0, \mathrm{MgCl}_{2} 1.0, \mathrm{CaCl}_{2}$ 1.35, HEPES 5.0, sodium cyanide 4, and BDM 20.0 to prevent muscle contracture. Buffer pH was adjusted with $\mathrm{KOH}$ or $\mathrm{HCl}$ to four different values ranging from 7.5 to 6.5. Such a calibration revealed a linear relation between $\mathrm{pH}$ and the fluorescence ratio (F495/F440) which was used to correct experimental ratios. All these ratios were calculated off-line after subtraction of the corresponding autofluorescence value at each wavelength as follows: (fluorescence at $495 \mathrm{~nm}$-autofluorescence at $495 \mathrm{~nm}$ ) / (fluorescence at 440 $\mathrm{nm}$-autofluorescence at $440 \mathrm{~nm}$ ). All pharmacological interventions were started 20 minutes before the ammonium prepulse and kept for the entire protocol.

\section{Western Blotting}

Papillary muscles were homogenized in RIPA buffer (Santa Cruz Biotechnology, sc-24948) with protease inhibitors cocktail, PMSF and sodium orthovanadate. After a brief centrifugation the supernatant was kept and protein concentration determined by the Bradford method (Bio-Rad dye reagent) as described by the manufacturer with BSA as a standard. Samples were denatured and equal amounts of protein were subjected to PAGE and electrotransferred to PVDF membranes. Membranes were then blocked with non-fat dry milk (5 \% in T-TBS) and incubated overnight with the specific antibodies against: phospho-ERK1 and ERK2, namely p-ERK1/2 (Santa Cruz Biotechnology, sc-16982), phospho-p90 RSK (Cell Signaling \#9341), phospho-p38-MAPK (Millipore, MABS64), phospho-(Ser16)-Phospholamban (Bradilla, A010-12), phosphoHSP27 (Santa Cruz Biotechnology, sc-166693) and DUSP6 (G-4) (Santa Cruz Biotechnology, sc-137246).

To determine NHE-1 phosphorylation at serine 703, the samples were immunoprecipitated using the NHE-1 polyclonal antibody and then subjected to PAGE, electrotransferred, blocked and incubated with anti-phospho-14-3-3 binding motif antibody (Cell Signaling, \#9601) as previously described [14].

To normalize the amount of the phosphorylated proteins the membranes were stripped and probed with an antibody that detects GAPDH (Millipore, MAB374), NHE-1 (Santa Cruz Biotechnology, sc-28758) or $\mathrm{Na}^{+} / \mathrm{K}^{+}$-ATPase $\alpha$ (H-3) (Santa Cruz Biotechnology, sc-48345).

Peroxidase-conjugated anti-rabbit (NA934, GE Healthcare Life Sciences) and anti-mouse (NA931, GE Healthcare Life Sciences) were used as secondary antibodies and bands were visualized using the Immobilon Western chemiluminescent HRP substrate (Millipore, WBKLS0100). Autoradiograms were analyzed by densitometry (Scion Image).

\section{RNA measurements by RT-PCR}

Total RNA was isolated from the homogenized papillary muscles using the RNeasy kit (Qiagen, Valencia, CA, USA) according to the manufacturer's instructions. RNA $(0.8 \mu \mathrm{g})$ was reverse-transcribed using the Omniscript RT kit (Qiagen). A dilution of the resulting cDNA was used for quantifying the relative content of mRNA by real-time PCR (iCycler iQ Real-Time PCR Detection System, Bio-Rad) using appropriate primers and SYBR Green as a fluorescent probe. The following primers, designed using Primer3 software, were used: 18S: forward primer 5-AGTCCCTGCCCTTTGTACAC-3; reverse primer 5-CCGAGGGCCTCACTAAACC-3; DUSP6: forward primer 5-CTGTGCCAAGGACTCTACTAAC-3; reverse primer 5-CAAACAGATTGGGCAGATTGG-3; DUSP7: forward primer 5-TCTCTCTCTCTCTCTCTCTCTCT -3; reverse primer 5-TGGCCACCCTCTGATATACT-3. PCR reactions were performed with TaqDNA polymerase (Invitrogen). Fluorescence data were acquired at the end of extension. A melt analysis was run for all of the products to determine the specificity of the amplification. The cycle threshold values for each gene were measured and calculated by computer software (iCycler IQ OSS, version 3.0a, Bio-Rad). The cycle threshold values for each gene were normalized by those of GAPDH in the same sample. For each experiment of real-time RT-PCR we obtained a melting curve of the amplified gene to confirm that only one product was amplified in each case with no primer-dimer formation. 


\section{Cellular Physiology Cell Physiol Biochem 2019;52:172-185 \\ \begin{tabular}{ll|l} 
and Biochemistry 10.33594/000000012 & O 2019 The Author(s). Published by \\
Published online: 28 February 2019 & Cell Physiol Biochem Press GmbH\&Co. KG
\end{tabular} \\ Zavala et al.: Myocardial Stretch and p38-MAPK}

Chemicals

All drugs used in the present study were analytical reagent. SB202190 and SB202474 were respectively purchased from Sigma (Cat \#S7067) and Santa Cruz Biotechnology (Cat \#222290). The 10 $\mu \mathrm{mol} / \mathrm{L}$ concentration used in this study was selected based on previous reports $[17,18]$, and diluted in DMSO (final concentration $0.1 \%$ ).

Statistics

Data are expressed as mean \pm SEM. One-way ANOVA followed by Student-Newman-Keuls test was used to compare data. A p value $<0.05$ was considered statistically significant.

\section{Results}

SFR development and p38-MAPK inhibition

As an initial approach to identify whether p38-MAPK influences the SFR, the contractile response to stretch of isolated rat papillary muscles was compared in the absence and presence of the p38-MAPK inhibitor SB202190 (10 $\mu \mathrm{mol} / \mathrm{L})$ or its inactive analogous, SB202474 $(10 \mu \mathrm{mol} / \mathrm{L})$. The typical biphasic force response of a muscle suddenly stretched from 92 to $98 \%$ of its maximal length is shown in Fig. 1A. The immediate increase in force is the expression of the well-known Frank-Starling mechanism, while the gradually developing one that follows, is the SFR. When the muscles were pre-incubated with the p38-MAPK inhibitor the SFR was significantly increased (Fig. 1B and 1D). Not significant changes in relaxation time were observed under p38-MAPK inhibition [time to half relaxation (msec), measured immediately and after 5 minutes of stretch, from $93 \pm 4$ to $93 \pm 5, n=16$ in control Stretch; and from $100 \pm 6$ to $102 \pm 6, n=21$ in Stretch+SB]. This finding indirectly suggests that myofilament responsiveness to calcium might not be responsible for the observed increase in force. Muscles pre-treated with the inactive analogous developed a SFR not different from untreated controls (Fig. 1C and 1D). Therefore, these results clearly indicate that p38-MAPK negatively regulates the SFR development.

\section{p38-MAPK activation after stretch}

In order to confirm that p38-MAPK is certainly activated by myocardial stretch, new experiments were performed in which papillary muscles were frozen 5 minutes after myocardial stretch and processed for phospho-p38-MAPK detection. We found a significant increase in p38-MAPK phosphorylation after stretch (Fig. 2A), effect that was precluded by pre-treatment with the p38-MAPK inhibitor (Fig. 2A). Moreover, to corroborate the activation of p38-MAPK after stretch, the phosphorylation level of HSP27, a well-known downstream target of p38-MAPK, was determined. Myocardial stretch induced a significant increase in HSP27 phosphorylation as depicted in Fig. 2B. Interestingly, muscles pre-treated with SB202190 did not show significant changes in HSP27 phosphorylation, while those pre-incubated with the inactive analogous SB202474 behaved like controls.

\section{NHE-1 phosphorylation and activity}

Since a phosphorylation-mediated increase in NHE-1 activity is a key factor in the chain of events leading to the SFR [19-23], we evaluated whether p38-MAPK inhibition had an effect upon this parameter. To this purpose, papillary muscles were frozen 5 minutes after myocardial stretch and processed for NHE-1 phosphorylation quantitation. As we have previously reported [14], myocardial stretch promoted a significant increase in NHE-1 phosphorylation at serine 703 (Fig. 3A). Interestingly, this increase was even greater in the presence of SB202190 (Fig. 3A), suggesting that p38-MAPK was probably limiting ERK1/2p90RSK activation and therefore NHE-1 phosphorylation/activation. 
Fig. 1. SFR and p38-MAPK inhibition. A: Original force record from a rat papillary muscle stretched from 92 to $98 \%$ of $L$ max showing the classical "two-phase" response. Individual traces corresponding to the points indicated in the upper panel with "a" (before stretch), "b" (immediately after stretch) and "c" (5 minutes after stretch) are shown. B: Same as A, but from a muscle preincubated with the p38MAPK inhibitor, SB202190 ("St+SB"), where it can be appreciated a greater SFR. C: same as A, but from a muscle pre-incubated with the SB202190 inactive analogous, SB202474 ("St+IA"). D: Averaged results after 5 minutes of stretch under all experimental conditions, expressed as a percentage of the initial rapid phase. Inset: The original force record shown in panel "A" was used to identify the characteristic two phases increase in force following myocardial stretch, as well as the calculation used to express the SFR in percentage form: "1" indicates the initial rapid phase generated immediately after stretch

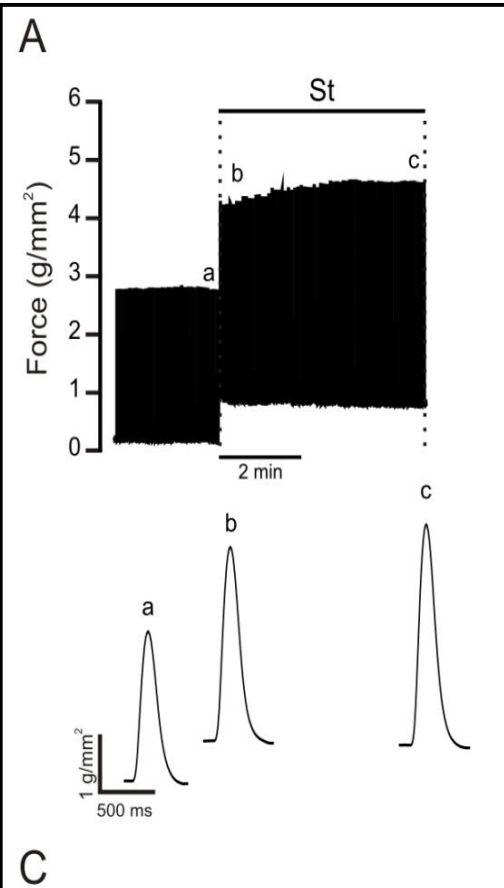

B
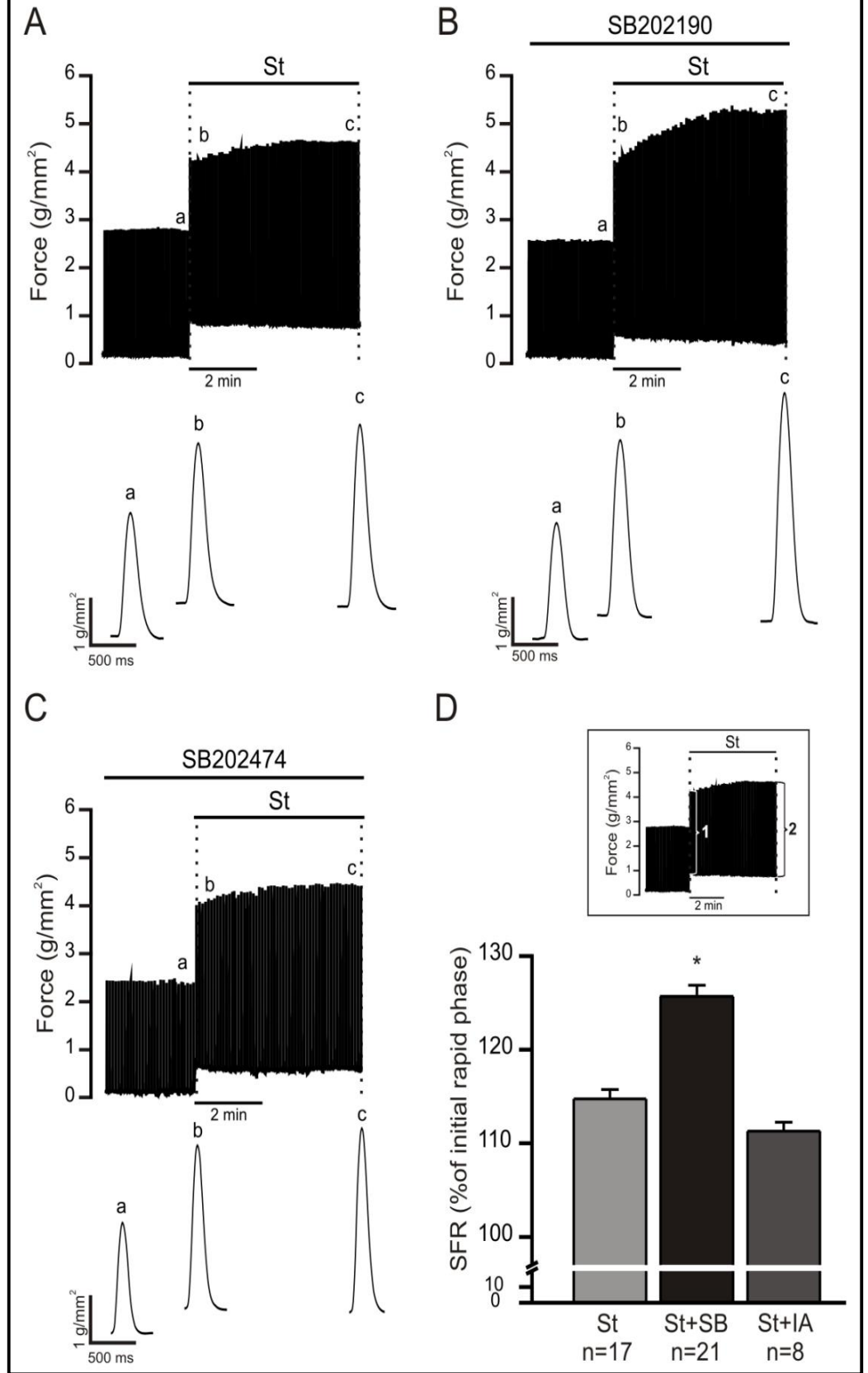
and considered as $100 \%$;

"2" highlights the SFR observed at 5 minutes of stretch. The final magnitude of the SFR was calculated as (2 * $100 \%) / 1$ * $^{*}$ indicates $\mathrm{p}<0.05$ vs. all other groups.

Since intracellular acidosis is the main stimulus for NHE- 1 activation and previous studies by Zheng et al. [24] have reported acidosis-mediated-p38-MAPK activation, NHE1 activity was evaluated by comparing $\mathrm{pH}_{\mathrm{i}}$ recovery from an ammonium prepulse-induced acute acid load in the absence and presence of the p38-MAPK inhibitor SB202190 or its inactive analogous, SB202474. Interestingly, our results revealed a significant increase in the maximal NHE-1-mediated proton efflux after the acidic challenge in the presence of SB202190 (Fig. 3B). In contrast, that effect was absent in muscles pre-treated with SB202474, the inactive analogous of SB202190 (Fig. 3B), confirming that p38-MAPK negatively regulates NHE-1 activity. 


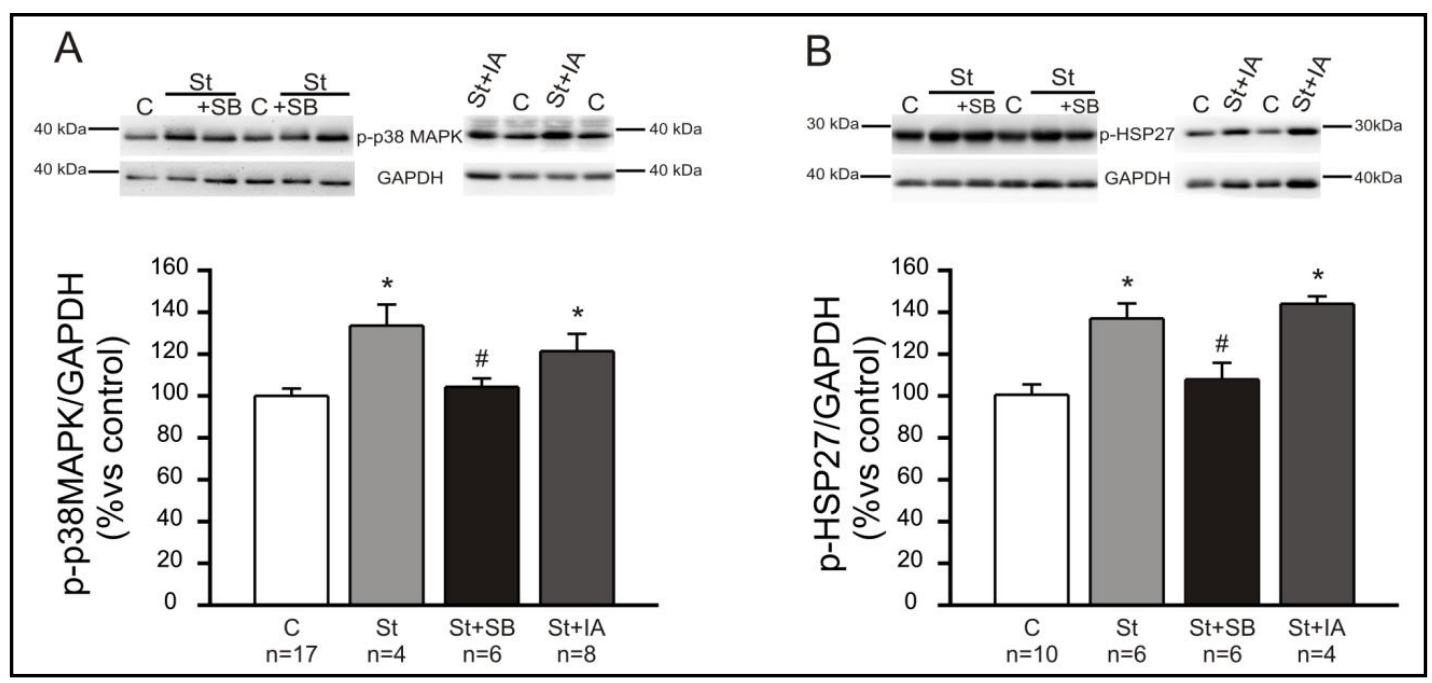

Fig. 2. p38-MAPK activation after myocardial stretch. A: Stretch ("St") significantly increased p38-MAPK phosphorylation (p-p38-MAPK) compared to the non-stretched control ("C"). As it can be appreciated, this effect was cancelled when the muscles were pre-treated with the p38-MAPK inhibitor SB202190 ("St+SB"), but not by SB202474, the inactive analogous of SB202190 ("St+IA"). B: Five minutes of stretch significantly increased HSP27 phosphorylation, a known downstream target of p38-MAPK. This effect was blunted by the p38-MAPK inhibitor ("St+SB"), but not by the inactive analogous ("St+IA"), demonstrating that p38MAPK is activated by the stretch. Bar graphs depict averaged data. Representative blots are shown on top of the bars. Importantly, neither SB202190 nor its inactive analogous (SB202474) alone modified basal p-p38-MAPK $(105 \pm 4 \%$ of control, $n=6$ and $102 \pm 4 \%$ of control, $n=4$, respectively) or p-HSP27 $(88 \pm 6 \%$ of control, $n=4$ and $98.5 \pm 12 \%$ of control, $n=4$ ). * indicates $\mathrm{p}<0.05$ vs control (non-stretched) and \# indicates $\mathrm{p}<0.05$ vs. St and St+IA.

\section{Crosstalk between p38-MAPK and ERK1/2-p90RSK after myocardial stretch}

Considering that ERK1/2 and p90RSK lead to NHE-1 activation during the SFR [14, 25], we explore the effect of p38-MAPK inhibition on the phosphorylation state of these kinases after stretch. Fig. 4 shows that myocardial stretch induced a greater increase in ERK1/2 and p90RSK phosphorylation when p38-MAPK was inhibited, consistent with the increase in NHE-1 phosphorylation described before (Fig. 3A). ERK1/2 and p90RSK phosphorylation level in muscles pre-treated with SB202474 was similar to that observed in untreated controls (Fig. 4). These results support the notion of a counterbalancing effect of p38-MAPK and ERK1/2 - p90RSK on NHE-1 phosphorylation during myocardial stretch.

\section{Phospholamban phosphorylation after stretch}

As we already mentioned, it has been reported that pharmacological inhibition of p38-MAPK strengthens the positive inotropic effect induced by ET-1 in isolated perfused rat hearts by increasing PLN phosphorylation at serine 16 [10]. Since ET is involved in the signaling pathway triggered by myocardial stretch (for review see [1]), we thought to explore whether this mechanism contributes to the bigger SFR observed under p38-MAPK inhibition. In contrast to this possibility, PLN phosphorylation at serine 16 was unaffected by myocardial stretch either in the absence or presence of SB202190 (Fig. 5), excluding its participation in the SFR.

\section{Up-regulation of DUSP6 after myocardial stretch}

To get further insight into the mechanism underlying the increase in the stretchinduced phosphorylation of ERK1/2, p90RSK and NHE-1 under p38-MAPK inhibition, we explored the putative involvement of DUSP6 and DUSP7, dual phosphatases that specifically dephosphorylates cytosolic ERK1/2 [26]. Fig. 6A shows a significant increase in DUSP6 mRNA abundance after $5 \mathrm{~min}$ of stretch, effect that was prevented by SB202190. In agreement, five 
Fig. 3. NHE-1 activation. A: In agreement with what we have shown previously [14], NHE-1 phosphorylation at serine 703 (estimated by a phospho-Ser 14-3-3 binding motif antibody, "p-14-3-3-BM") was significantly increased by myocardial stretch ("St") compared to non-stretched controls ("Control"), as shown in the original western blots (top) and the averaged data (bottom). Interestingly, this effect was magnified in stretched muscles pre-treated with the p38-MAPK inhibitor, SB202190 ("St+SB"). SB alone did not modify basal NHE-1 phosphorylation $(92 \pm 6 \%$ of control, $\mathrm{n}=3$ ). B: Consistently, p38-MAPK inhibition ("SB") significantly increased NHE-1mediated $\mathrm{H}^{+}$efflux $\left(\mathrm{J}_{\mathrm{H}}\right)$ after an acidic challenge (ammonium prepulse), effect that was not observed in muscles pre-treated with the inactive analogous of SB202190, SB202474 (IA). Typical $\mathrm{pH}_{\mathrm{i}}$ recordings (top) and averaged initial (maximal) $\mathrm{H}^{+}$efflux (bottom) under all experimental conditions are shown. For the sake of comparison, the best exponential fit for each $\mathrm{pH}_{\mathrm{i}}$ recovery from acidosis was superimposed. * indicates $\mathrm{p}<0.05$ vs non-stretched Control, ** indicates $\mathrm{p}<0.05$ vs. St, and \# indicates $\mathrm{p}<0.05$ vs. Control acidosis (CA) and IA.
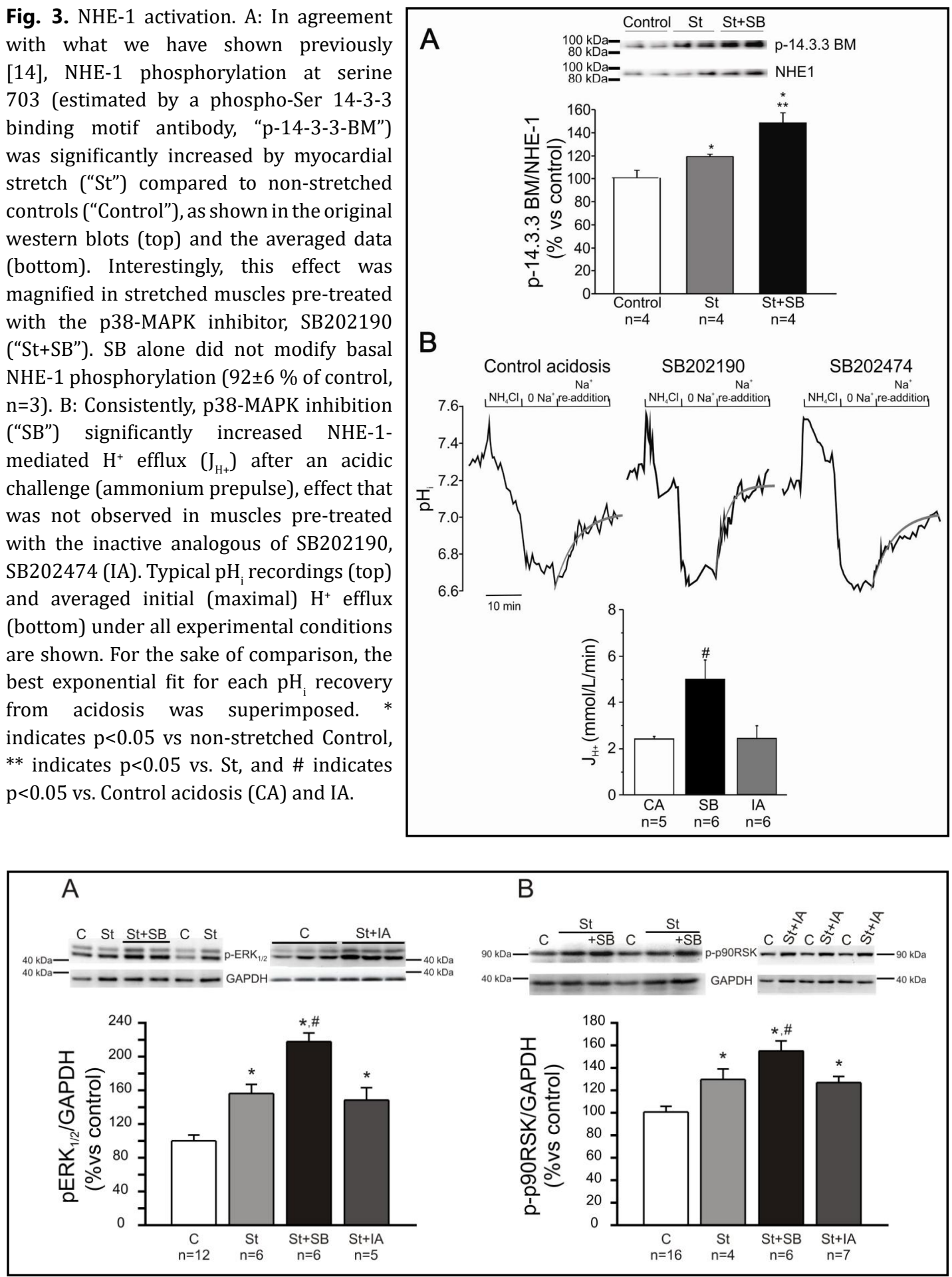

Fig. 4. ERK1/2 and p90RSK phosphorylation after stretch. As reported previously [25], myocardial stretch (St) significantly increased ERK1/2 (A) and p90RSK (B) phosphorylation compared to non-stretched controls ("Control" or "C"). In the presence of the p38-MAPK inhibitor SB202190 ("SB"), myocardial stretch promoted a greater increase in ERK1/2 and p90RSK phosphorylation, effect that was not observed in muscles pre-treated with the inactive analogous SB202474 ("St+IA"). SB and IA alone did not modify the phosphorylation of either ERK1/2 (90 $\pm 9 \%$ of control, $n=5$ and $97 \pm 6 \%$ of control, $n=5$, respectively) or p90RSK ( $105 \pm 4 \%$ of control, $n=5$ and $93 \pm 8 \%$ of control, $n=4$, respectively). ${ }^{*}$ indicates $\mathrm{p}<0.05$ vs. control, \# indicates $\mathrm{p}<0.05$ vs. St and St $+\mathrm{IA}$. 
Fig. 5. Phospholamban phosphorylation after stretch. Phospholamban (PLN) phosphorylation at serine 16 was unaffected by myocardial stretch either in the absence ("St") or presence ("St+SB") of SB202190 compared to non-stretched controls ("C"). Representative blots (top) and averaged data (bottom) under all experimental conditions are shown. PLN phosphorylation was expressed as \% of its maximal phosphorylation state induced by $100 \mathrm{nmol} / \mathrm{L}$ isoproterenol ("Iso"). * indicates $\mathrm{p}<0.05$ vs. all other groups.

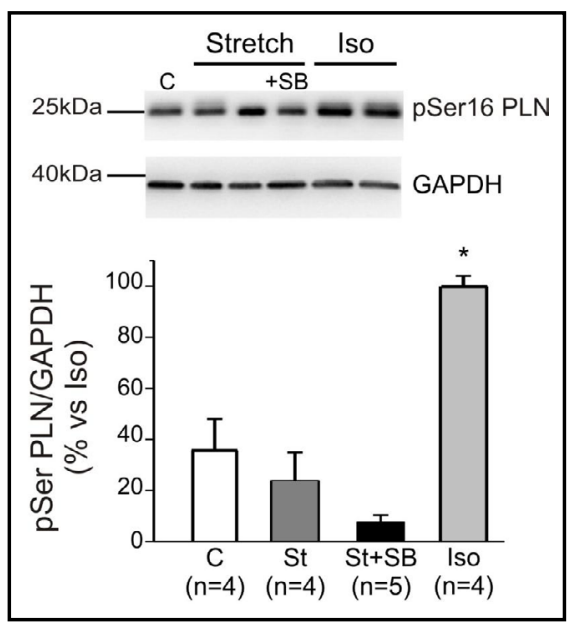

Fig. 6. Up-regulation of DUSP6 after myocardial stretch. Five minutes of myocardial stretch ("St") promotes a significant increase in mRNA (A) and protein (B) expression of DUSP6, compared to non-stretched controls ("C"), effects that were precluded by the p38-MAPK inhibitor SB202190 ("St+SB"). In contrast, not significant changes in DUSP7 mRNA expression was found either in the absence or presence of SB202190 (C).* indicates $\mathrm{p}<0.05$ vs. Control ("C"), \# indicates p $<0.05$ vs. St.

minutes of myocardial stretch promoted a significant increase in the protein expression of DUSP6, compared to non-stretched controls, effect that was abrogated by the p38-MAPK inhibitor SB202190 (Fig. 6B). These results suggest that p38-MAPK activation by stretch induces the up-regulation of DUSP6. In contrast, DUSP7 appears not to be involved in the mechanism triggered by myocardial stretch as shown in Fig. 6C. It is important to keep in mind that the activity of these phosphatases depends on its level of expression [2628].

\section{Discussion}

Our current data provide strong evidence to include the activation of p38-MAPK and DUSP6 as novel steps in the complex mechanism triggered by myocardial stretch and leading to the SFR. The major findings of our study are that p38-MAPK inhibition strengthens the stretch-stimulated ERK1/2-p90RSK and NHE1 phosphorylation and activities, consequently promoting a greater SFR. We propose that cardiac stretch promotes a p38-MAPK-mediated increase in

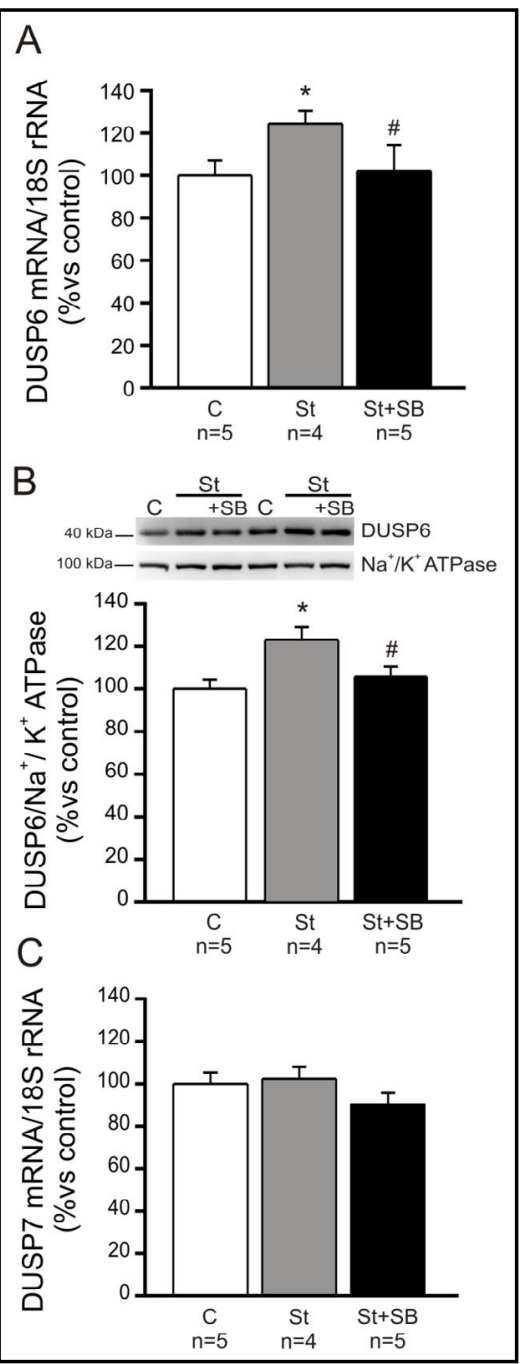
DUSP6 activity, restricting ERK1/2-p90RSK and NHE1 phosphorylation/activation and therefore limiting the SFR development. Conversely, under p38-MAPK inhibition the prevention of DUSP6 upregulation would allow a larger increase in ERK1/2 phosphorylation, which determines a greater activity of p90RSK and NHE-1, leading to a bigger SFR. 


\section{Cellular Physiology Cell Physiol Biochem 2019;52:172-185 \\ \begin{tabular}{ll|l} 
and Biochemistry & $\begin{array}{l}\text { DOl: 10.33594/000000012 } \\
\text { Published online: } 28 \text { February } 2019\end{array}$ & $\begin{array}{l}\text { O } 2019 \text { The Author(s). Published by } \\
\text { Cell Physiol Biochem Press GmbH\&Co. KG }\end{array}$ \\
\cline { 2 - 3 }
\end{tabular} \\ Zavala et al.: Myocardial Stretch and p38-MAPK}

Previous reports by Liao et al. [5] and by Kockskamper et al. [29] support the notion that p38-MAPK activation negatively regulates cardiac contractility by decreasing myofilament $\mathrm{Ca}^{2+}$ responsiveness. The latter study is of particular interest for us since the authors described a greater SFR to stretch in human trabeculae when the activation of p38-MAPK was prevented by SB203580, a different inhibitor from the one used herein. Furthermore, they also described an increase in basal developed force after p38-MAPK inhibition, suggesting that this kinase regulates cardiac contractility not only after stretch but also under basal conditions. Although the authors stated that the precise mechanism underlying these effects was still unknown, the fact that the positive inotropic effect detected after p38-MAPK inhibition was associated with a prolonged relaxation time, leaded them to tentatively assign a role to increased myofilament $\mathrm{Ca}^{2+}$ sensitivity as mentioned above. However, our current results do not appear to support their findings. In fact, the finding of an increased NHE-1 phosphorylation/activity under p38-MAPK inhibition (Fig. 3) allows us to suggest that a greater increase in intracellular $\mathrm{Ca}^{2+}$, as a consequence of NHE-1-dependent intracellular $\mathrm{Na}^{+}$overload, would be the main responsible for the larger SFR detected under these experimental conditions. Furthermore, although our data do not allow to completely exclude changes in myofilament $\mathrm{Ca}^{2+}$ responsiveness contributing to the increase in SFR, the absence of changes in the relaxation time during the SFR appears to reject this possibility. On the other hand, the fact that p38-MAPK inhibition failed to alter baseline contractility, suggests that p38-MAPK does not regulate basal contractility in rat papillary muscles.

The most relevant group of protein phosphatases in mammalian cells that specifically regulates MAPKs activity is the dual specificity MAPK phosphatases, (MKPs or DUSPs) [30]. The main characteristic of several DUSPs is their regulation at the level of gene expression following stress or mitogen stimulation, providing a negative feedback to limit the extent and duration of MAPK signaling [26-28]. Once expressed, the DUSPs are constitutively active and capable of directly binding to the activation loop in MAPKs, resulting in dephosphorylation. Each DUSPs differs with respect to the subcellular localization, tissue expression, or specificity for each MAPK [26-28]. DUSP6 is a member of the large family of MAPK phosphatases that bind with varying specificity to ERK1/2, JNK1/2, p38-MAPK, ERK5, and presumably ERK3/4 $[26,27]$. DUSP 6 is known to form a complex with ERK2 through a series of amino acids that are conserved in ERK1, but not ERK5 or ERK3/4, suggesting that it is highly specific for ERK1/2. In Dusp $\%$ mice, Maillet et al. [31] showed an increase in basal ERK1/2 phosphorylation in several tissues, but no change in ERK5, p38-MAPK, or c-Jun N-terminal kinases activation. Even more importantly, Purcell et al. [32] generated transgenic mice overexpressing DUSP6 in the heart and showed a complete absence of ERK1/2 phosphorylation at baseline or after any applied stress stimulus; nevertheless ERK5, p38-MAPK, and JNK phosphorylation were unaffected [32]. Although the experimental evidence indicates that DUSP6 only affects ERK1/2 within the greater MAPK family, we cannot rule out the possibility that it could affect other proteins implicated in the SFR development.

In the present study, we did not explore the mechanism by which myocardial stretch up-regulates DUSP6. It has been described that DUSP6 expression is induced in response to elevated ERK1/2 signalling as a feedback regulator [33]. Our current results do not appear to support this concept since when the stretch was performed under p38-MAPK inhibition (Fig. 4) we observed a greater increase in ERK1/2 without changes in DUSP6 expression, which was similar to that in non-stretched muscles (Fig. 6A). Therefore, our data strongly suggests a p38-MAPK-mediated increase in DUSP6 expression after myocardial stretch.

Modulation of ERK1/2 activity by p38-MAPK has been previously described in different tissues [11, 34]. Furthermore, it has been shown that specific activation of p38-MAPK by overexpression of a constitutively active MAPK kinase-3b inhibits ERK1/2 [35]. Our result showing p38-MAPK-dependent inhibition of ERK1/2 phosphorylation following myocardial stretch is consistent with these findings.

A previous work reported the direct phosphorylation of NHE-1 between amino acids 625 and 747 by p38-MAPK [11]. The authors speculated that this phosphorylation might either 


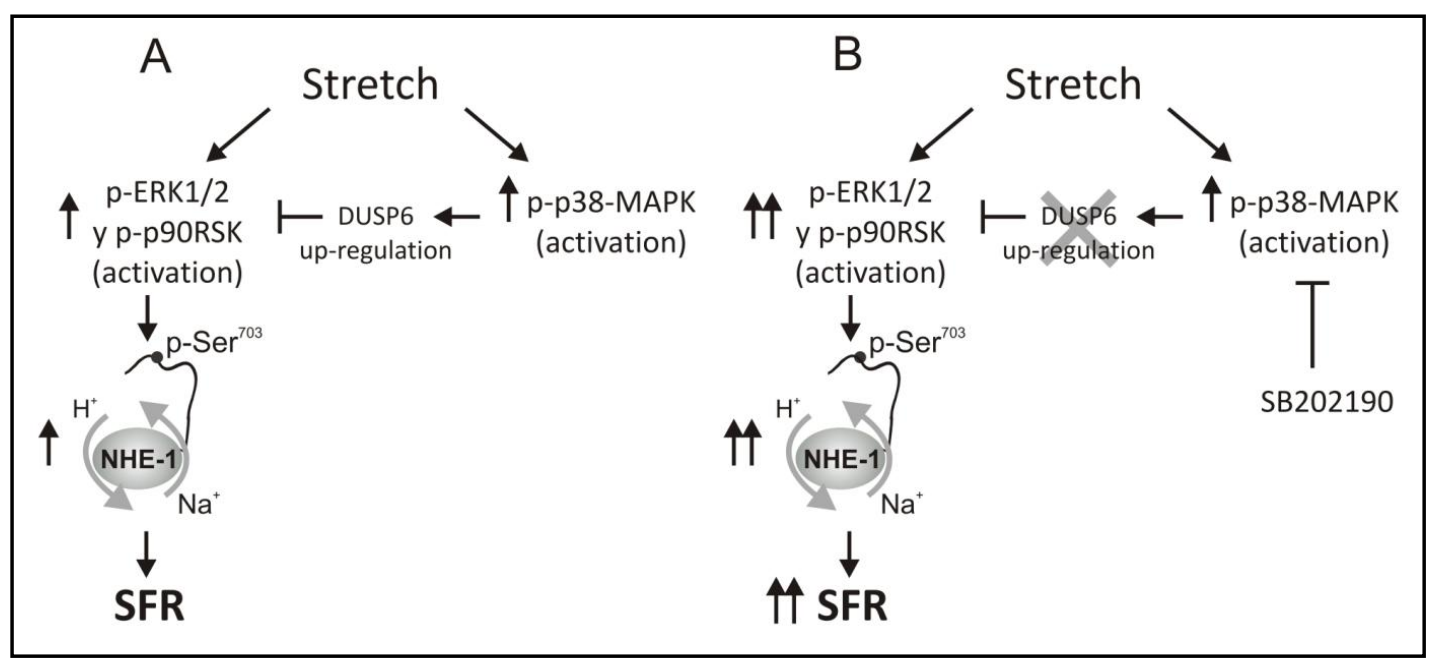

Fig. 7. Schematic representation of the proposed mechanism, based on our current and previous findings. Under control conditions (A), myocardial stretch triggers p38-MAPK activation and DUSP6 up-regulation, therefore restricting the increase in ERK1/2-p90RSK and NHE-1 phosphorylation/activation [14, 25], with the consequent limitation of the SFR amplitude. Prevention of p38-MAPK activation (B) precludes DUSP6 up-regulation after stretch allowing a higher phosphorylation of ERK1/2-p90RSK and consequently of NHE1 , leading to a greater increase in force during the SFR development.

induce a conformational change that inhibits transport activity, or prevent the interaction with other regulatory molecules crucial for transport activity. Herein, we measured NHE1 phosphorylation at the residue serine 703, a documented p90RSK target that does not appear to be phosphorylated by p38-MAPK [12], detecting an increased phosphorylation of this site when myocardial stretch was performed under p38-MAPK inhibition.

Finally, in isolated perfused rat hearts, Ruskoaho et al. [10] were able to show that the positive inotropic effect of ET-1, a known mediator of the SFR to stretch, can be diminished by pharmacological inhibition of the NHE-1. Interestingly, they also showed that the increase in force could be potentiated by p38-MAPK inhibition, being the positive inotropic effect due to enhanced SERCA2a activity as consequence of increased PLN phosphorylation [10]. Despite the differences in the experimental conditions, as well as the potent vasoconstrictor effect of ET-1 in the perfused heart, the demonstrated crucial role of NHE-1 activation in the peptide-triggered positive inotropic response is in line with previous findings of our group in isolated papillary muscles $[21,36]$. However, we were not able to detect significant changes in PLN phosphorylation following myocardial stretch under p38-MAPK inhibition (Fig. 5), reinforcing the notion that the sarcoplasmic reticulum does not participate in the development of the SFR, as reported by others [37-39].

\section{Conclusion}

In conclusion, the present study demonstrates that p38-MAPK activation after stretch negatively regulates the SFR. The proposed underlying mechanism is that myocardial stretch activates p38-MAPK up-regulating DUSP6 that in turn limits ERK1/2-p90RSK and NHE-1 phosphorylation/activation. A schematic representation of the proposed mechanism is depicted in Fig. 7. 


\section{Cellular Physiology Cell Physiol Biochem 2019;52:172-185 \begin{tabular}{ll|l} 
and Biochemistry 10.33594/000000012 & O 2019 The Author(s). Published by \\
Published online: 28 February 2019 & Cell Physiol Biochem Press GmbH\&Co. KG
\end{tabular} \\ Zavala et al.: Myocardial Stretch and p38-MAPK}

\section{Acknowledgements}

This work was supported in part by PICT 2014-1933 from Agencia Nacional de Promoción Científica y Tecnológica de Argentina to Dr. MC Villa-Abrille, by PICT 2012-2396 and 2016-2289 from Agencia Nacional de Promoción Científica y Tecnológica de Argentina to Dr. NG Pérez, by PIP2015-0750 from CONICET to Dr. NG Pérez, and by Beca Estímulo 2013 from Sociedad Argentina de Hipertensión Arterial to Dr. MC Villa-Abrille. Maite R. Zavala and Romina G. Díaz contributed equally to this article. We specially thank Omar Castillo for technical assistance.

\section{Disclosure Statement}

The authors declare to have no competing interests.

\section{References}

- 1 Cingolani HE, Perez NG, Cingolani OH, Ennis IL: The Anrep effect: 100 years later. Am J Physiol Heart Circ Physiol 2013;304:H175-H182.

- 2 Baines CP, Molkentin JD: STRESS signaling pathways that modulate cardiac myocyte apoptosis. J Mol Cell Cardiol 2005;38:47-62.

- 3 Kerkela R, Force T: p38 mitogen-activated protein kinase: a future target for heart failure therapy? J Am Coll Cardiol 2006;48:556-558.

4 Rose BA, Force T, Wang Y: Mitogen-activated protein kinase signaling in the heart: angels versus demons in a heart-breaking tale. Physiol Rev 2010;90:1507-1546.

5 Liao P, Wang SQ, Wang S, Zheng M, Zheng M, Zhang SJ, Cheng H, Wang Y, Xiao RP: p38 Mitogen-activated protein kinase mediates a negative inotropic effect in cardiac myocytes. Circ Res 2002;90:190-196.

6 Auger-Messier M, Accornero F, Goonasekera SA, Bueno OF, Lorenz JN, van Berlo JH, Willette RN, Molkentin JD: Unrestrained p38 MAPK activation in Dusp1/4 double-null mice induces cardiomyopathy. Circ Res 2013;112:48-56.

7 Bellahcene M, Jacquet S, Cao XB, Tanno M, Haworth RS, Layland J, Kabir AM, Gaestel M, Davis RJ, Flavell RA, Shah AM, Avkiran M, Marber MS: Activation of p38 mitogen-activated protein kinase contributes to the early cardiodepressant action of tumor necrosis factor. J Am Coll Cardiol 2006;48:545-555.

8 Kerkela R, Ilves M, Pikkarainen S, Tokola H, Ronkainen VP, Majalahti T, Leppaluoto J, Vuolteenaho 0, Ruskoaho H: Key roles of endothelin-1 and p38 MAPK in the regulation of atrial stretch response. Am J Physiol Regul Integr Comp Physiol 2011;300:R140-R149.

9 Vahebi S, Ota A, Li M, Warren CM, de Tombe PP, Wang Y, Solaro RJ: p38-MAPK induced dephosphorylation of alpha-tropomyosin is associated with depression of myocardial sarcomeric tension and ATPase activity. Circ Res 2007;100:408-415.

10 Szokodi I, Kerkela R, Kubin AM, Sarman B, Pikkarainen S, Konyi A, Horvath IG, Papp L, Toth M, Skoumal R, Ruskoaho H: Functionally opposing roles of extracellular signal-regulated kinase 1/2 and p38 mitogenactivated protein kinase in the regulation of cardiac contractility. Circulation 2008;118:1651-1658.

11 Kusuhara M, Takahashi E, Peterson TE, Abe J, Ishida M, Han J, Ulevitch R, Berk BC: p38 Kinase is a negative regulator of angiotensin II signal transduction in vascular smooth muscle cells: effects on $\mathrm{Na}+\mathrm{H}+$ exchange and ERK1/2. Circ Res 1998;83:824-831.

12 Takahashi E, Abe J, Gallis B, Aebersold R, Spring DJ, Krebs EG, Berk BC: p90(RSK) is a serum-stimulated $\mathrm{Na}+\mathrm{H}+$ exchanger isoform-1 kinase. Regulatory phosphorylation of serine $703 \mathrm{of} \mathrm{Na} / \mathrm{H}+$ exchanger isoform-1. J Biol Chem 1999;274:20206-20214.

- 13 National Research Council (US) Committee for the Update of the Guide for the Care and Use of Laboratory Animals: Guide for the Care and Use of Laboratory Animals, 8th ed. Washington (DC), The National Academies Press, 2011. 


\section{Cellular Physiology Cell Physiol Biochem 2019;52:172-185 \begin{tabular}{l|l|l}
\cline { 2 - 3 } DOl: 10.33594/000000012 & (c) 2019 The Author(s). Published by
\end{tabular} and Biochemistry Published online: 28 February 2019 Cell Physiol Biochem Press GmbH\&Co. KG \\ Zavala et al.: Myocardial Stretch and p38-MAPK}

14 Villa-Abrille MC, Caldiz CI, Ennis IL, Nolly MB, Casarini MJ, Chiappe de Cingolani GE, Cingolani HE, Perez NG: The Anrep effect requires transactivation of the epidermal growth factor receptor. J Physiol 2010;588:1579-1590.

- 15 Diaz RG, Nolly MB, Massarutti C, Casarini MJ, Garciarena CD, Ennis IL, Cingolani HE, Perez NG: Phosphodiesterase 5A inhibition decreases NHE-1 activity without altering steady state $\mathrm{pH}(\mathrm{i})$ : role of phosphatases. Cell Physiol Biochem 2010;26:531-540.

- 16 Camilion de Hurtado MC, Ennis IL, Perez NG, Chiappe de Cingolani GE, Morgan P, Cingolani HE: Upregulation of myocardial $\mathrm{Na}+/ \mathrm{H}+$ exchanger induced by chronic treatment with a selective inhibitor. J Mol Cell Cardiol 2002;34:1539-1547.

- 17 Lv XC, Zhou HY: Resveratrol protects H9c2 embryonic rat heart derived cells from oxidative stress by inducing autophagy: role of p38 mitogen-activated protein kinase. Can J Physiol Pharmacol 2012;90:655662.

18 Omar MA, Verma S, Clanachan AS: Adenosine-mediated inhibition of 5'-AMP-activated protein kinase and p38 mitogen-activated protein kinase during reperfusion enhances recovery of left ventricular mechanical function. J Mol Cell Cardiol 2012;52:1308-1318.

- 19 Alvarez BV, Perez NG, Ennis IL, Camilion de Hurtado MC, Cingolani HE: Mechanisms underlying the increase in force and $\mathrm{Ca}(2+)$ transient that follow stretch of cardiac muscle: a possible explanation of the Anrep effect. Circ Res 1999;85:716-722.

- 20 Cingolani HE, Perez NG, Aiello EA, de Hurtado MC: Intracellular signaling following myocardial stretch: an autocrine/paracrine loop. Regul Pept 2005;128:211-220.

- 21 Cingolani HE, Alvarez BV, Ennis IL, Camilion de Hurtado MC: Stretch-induced alkalinization of feline papillary muscle: an autocrine-paracrine system. Circ Res 1998;83:775-780.

- 22 Calaghan SC, White E: Contribution of angiotensin II, endothelin 1 and the endothelium to the slow inotropic response to stretch in ferret papillary muscle. Pflugers Arch 2001;441:514-520.

- 23 Luers C, Fialka F, Elgner A, Zhu D, Kockskamper J, von Lewinski D, Pieske B: Stretch-dependent modulation of $[\mathrm{Na}+] \mathrm{i},[\mathrm{Ca} 2+] \mathrm{i}$, and pHi in rabbit myocardium--a mechanism for the slow force response. Cardiovasc Res 2005;68:454-463.

24 Zheng M, Hou R, Xiao RP: Acidosis-induced p38 MAPK activation and its implication in regulation of cardiac contractility. Acta Pharmacol Sin 2004;25:1299-1305.

25 Caldiz CI, Garciarena CD, Dulce RA, Novaretto LP, Yeves AM, Ennis IL, Cingolani HE, Chiappe de Cingolani G, Perez NG: Mitochondrial reactive oxygen species activate the slow force response to stretch in feline myocardium. J Physiol 2007;584:895-905.

- 26 Theodosiou A, Ashworth A: MAP kinase phosphatases. Genome Biol 2002;3:REVIEWS3009.

- 27 Owens DM, Keyse SM: Differential regulation of MAP kinase signalling by dual-specificity protein phosphatases. Oncogene 2007;26:3203-3213.

- 28 Camps M, Nichols A, Arkinstall S: Dual specificity phosphatases: a gene family for control of MAP kinase function. FASEB J 2000;14:6-16.

- 29 Kockskamper A, von Lewinski D, Zhu D, Kockskamper J, Khafaga M, Schmidt AG, Post H, Pieske B: Relevance of stretch-induced phosphorylation of MAPK and p90rsk in human myocardium. Front Biosci (Elite Ed) 2013;5:883-892.

- 30 Keyse SM: Protein phosphatases and the regulation of mitogen-activated protein kinase signalling. Curr Opin Cell Biol 2000;12:186-192.

- 31 Maillet M, Purcell NH, Sargent MA, York AJ, Bueno OF, Molkentin JD: DUSP6 (MKP3) null mice show enhanced ERK1/2 phosphorylation at baseline and increased myocyte proliferation in the heart affecting disease susceptibility. J Biol Chem 2008;283:31246-31255.

32 Purcell NH, Wilkins BJ, York A, Saba-El-Leil MK, Meloche S, Robbins J, Molkentin JD: Genetic inhibition of cardiac ERK1/2 promotes stress-induced apoptosis and heart failure but has no effect on hypertrophy in vivo. Proc Natl Acad Sci U S A 2007;104:14074-14079.

- 33 Bermudez 0, Pages G, Gimond C: The dual-specificity MAP kinase phosphatases: critical roles in development and cancer. Am J Physiol Cell Physiol 2010;299:C189-C202.

- 34 Liu Q, Hofmann PA: Protein phosphatase 2A-mediated cross-talk between p38 MAPK and ERK in apoptosis of cardiac myocytes. Am J Physiol 2004;286:H2204-H2212. 


\section{Cellular Physiology Cell Physiol Biochem 2019;52:172-185}

\begin{tabular}{ll|ll} 
and Biochemistry & DO.33594/000000012 & O 2019 The Author(s). Published by \\
Published online: 28 February 2019 & Cell Physiol Biochem Press GmbH\&Co. KG
\end{tabular}

Zavala et al.: Myocardial Stretch and p38-MAPK

35 Westermarck J, Li SP, Kallunki T, Han J, Kahari VM: p38 mitogen-activated protein kinase-dependent activation of protein phosphatases 1 and 2A inhibits MEK1 and MEK2 activity and collagenase 1 (MMP-1) gene expression. Mol Cell Biol 2001;21:2373-2383.

- 36 Perez NG, Villa-Abrille MC, Aiello EA, Dulce RA, Cingolani HE, Camilion de Hurtado MC: A low dose of angiotensin II increases inotropism through activation of reverse $\mathrm{Na}(+) / \mathrm{Ca}(2+)$ exchange by endothelin release. Cardiovasc Res 2003;60:589-597.

37 Kentish JC, Wrzosek A: Changes in force and cytosolic Ca2+ concentration after length changes in isolated rat ventricular trabeculae. J Physiol 1998;506:431-444.

38 Bluhm WF, Lew WY: Sarcoplasmic reticulum in cardiac length-dependent activation in rabbits. Am J Physiol 1995;269:H965-H972.

39 Hongo K, White E, Orchard CH: Effect of stretch on contraction and the Ca2+ transient in ferret ventricular muscles during hypoxia and acidosis. Am J Physiol 1995;269:C690-C697. 